\title{
A Role of Cytokinin Transporter in Arabidopsis Immunity
}

\author{
Shuai Wang, ${ }^{1,2}$ Shu Wang, ${ }^{1}$ Qi Sun, ${ }^{3}$ Leiyun Yang, ${ }^{1}$ Ying Zhu, ${ }^{1,4}$ Yaping Yuan, ${ }^{2}$ and Jian Hua \\ ${ }^{1}$ Plant Biology Section, School of Integrative Plant Science, Cornell University, Ithaca, New York, 14853, U.S.A.; ${ }^{2}$ College of \\ Plant Science, Jilin University, Jilin, 130062, P.R. China; and ${ }^{3}$ Computational Biology Service Unit, Cornell University; ${ }^{4}$ Institute \\ of Virology and Biotechnology, Zhejiang Academy of Agricultural Sciences, Hangzhou, Zhejiang, P.R. China
}

Accepted 23 February 2017.

\begin{abstract}
The phytohormone cytokinin (CK) is not only essential for plant growth and development but also impacts plant immunity. A mutant screen in a constitutively active plant immune receptor mutant snc1 (suppressor of npr1, constitutive1) identified a suppressor mutation of $S N C 1$-induced defense responses in an ABC transporter coding gene ABCG14. ABCG14 transports $\mathrm{CK}$ from roots to the shoots, and the suppression of the $S N C 1$-mediated defense response by the loss of $A B C G 14$ is due to a deficiency of trans-zeatin $(t Z)$-type $C K$ in the shoot. In addition, exogenous application of the $t Z$-type CK enhances disease resistance associated with increased expression of the plant immune receptor gene $S N C 1$. Taken together, this study further established the role of $t \mathrm{Z}$-type $\mathrm{CK}$ in disease resistance and suggests a new intersection of CKs with plant immunity at the expression regulation of a plant immune receptor gene.
\end{abstract}

Plant immunity against pathogens occurs at multiple levels and there are at least two branches of defenses at the molecular level (Jones and Dangl 2006). One is triggered by the perception of pathogen-associated molecular patterns (PAMP) with transmembrane pattern recognition receptors, namely PAMP-triggered immunity (PTI). The other is triggered by the recognition of pathogen-secreted effectors by intracellular immune receptors or resistance (R) proteins, namely effector triggered immunity. Specific recognition of effectors by immune receptor $\mathrm{R}$ proteins leads to rapid and efficient defense responses, including a form of programmed cell death named hypersensitive response, which restricts the growth and spread of pathogens (Hammond-Kosack and Jones 1996).

Phytohormones function as intrinsic signals to regulate plant growth and development as they directly regulate cell division and cell expansion. They also respond to biotic and abiotic signals and mediate adaptive responses to environmental changes. With pathogen infection or herbivore attack, a number of hormones have altered levels and are shown to contribute to plant immunity (Bari and Jones 2009; Howe and Jander 2008;

Current address for Shuai Wang and Shu Wang: The State Key Laboratory of Crop Genetics and Germplasm Enhancement, Bioinformatics Center, Nanjing Agricultural University, Nanjing, 210095, P.R. China.

Current address for Ying Zhu: Institute of Virology and Biotechnology, Zhejiang Academy of Agricultural Sciences, Hangzhou, 310021, P.R. China.

Corresponding author: Jian Hua; E-mail: jh299@ @ornell.edu.

*The $\boldsymbol{e}$-Xtra logo stands for "electronic extra" and indicates that one supplementary table is published online.

๑) 2017 The American Phytopathological Society
Katagiri and Tsuda 2010; Pieterse et al. 2009). Salicylic acid (SA) and jasmonic acid (JA) are central players in defenses against biotrophic and necrotrophic pathogens, respectively (Browse 2009; Vlot et al. 2009). Other hormones, including ethylene (ET) (van Loon et al. 2006), gibberellins (GAs) (Navarro et al. 2008), abscisic acid (ABA) (Ton et al. 2009), auxins (Kazan and Manners 2009), brassinosteroids (Nakashita et al. 2003), and cytokinins (CKs) (Walters and McRoberts 2006), act as modulators of plant defense responses as well. ET is widely considered to cooperate with JA to induce necrotroph resistance while antagonizing SA-mediated biotroph resistance (Derksen et al. 2013). GA signaling acts as a negative regulator of resistance to the necrotrophic fungus Alternaria brassicicola, because the Arabidopsis mutants lacking four of the five GA signaling-repressing DELLA proteins showed enhanced susceptibility to the necrotroph pathogen (Achard et al. 2008; Navarro et al. 2008). ABA has multifaceted interaction with plant immunity (Ton et al. 2009), and ABA and SA affect each other at multiple steps from biosynthesis to signaling (Cao et al. 2011; de Torres Zabala et al. 2009; Jiang et al. 2010; Mang et al. 2012; Yasuda et al. 2008). Auxin has a negative role in pathogen resistance. During PTI, induction of the microRNA miR393 by the bacterial PAMP flagellin targets auxin receptor gene TIRl, leading to an enhancement of pathogen resistance by suppressing auxin signaling (Navarro et al. 2006).

CKs consist of a highly complex family of $N^{6}$-substituted adenine derivatives and chemically unrelated phenylurea-type regulatory molecules, such as trans-zeatin $(t Z), N^{6}-\left(\Delta^{2}\right.$-isopentenyl) adenine (iP), dihydrozeatin, and cis-zeatin. These compounds are active at the sites of their biosynthesis (Zhao 2008) as well as in distant tissues, in which they arrive via long-distance transport. ABCG14, an ABC-type (ATP-binding cassette) transporter, is shown to mediate the translocation of $t$ Z-type CK from roots to shoots through xylem and to regulate shoots growth (Ko et al. 2014; Zhang et al. 2014). CKs have long been known to be involved in plant-microbe interactions. Overaccumulation of CKs is associated with disease symptoms and morphological anomalies such as fasciation, senescence, and the formation of tumors, galls, and socalled green islands (Grant and Jones 2009). A dozen bacterial and fungal pathogens can either secrete CKs themselves, induce CK biosynthesis in plants, or both (Naseem et al. 2014; Siemens et al. 2006; Walters et al. 2008). In addition, pathogens may also activate plant CK signaling, leading to the suppression of plant immunity. HopQ1, an effector from Pseudomonas syringae induces the expression of type A Arabidopsis response regulator (ARR) genes in CK signaling to downregulate expression of the flagellin receptor gene FLS2 in PTI (Hann et al. 2014).

The levels of CKs are found to impact the strength of plant immunity. Low concentrations of the benzyl adenine (BA) CK enhanced susceptibility of Arabidopsis to the biotrophic pathogen Hyaloperonospora arabidopsidis, whereas high concentrations of BA enhanced resistance to the pathogen in Arabidopsis 
(Argueso et al. 2012). CK has a positive role in resistance to hemibiotrophic Pseudomonas syringae pv. tomato DC3000. The CK receptor mutant ahk2ahk3 and transgenic line overexpression of CK oxidases (CKXs) both exhibited enhanced susceptibility to $P$. syringae pv. tomato DC3000 (Choi et al. 2010). CK is shown to interact with SA to affect immunity. Synergistic interaction is found between CKs and SA in resistance to $P$. syringae pv. tomato DC3000, and a direct interaction was found between the CK-activated transcription factor ARR2 and the SA-response transcription factor TGA3 (TGA1A-related gene 3) (Choi et al. 2010, 2011). Overexpression of a CK response factor 2 (AtCRF2) enhances SA biosynthesis and leads to autoimmune response in Arabidopsis (Kwon 2016). On the other hand, SA is found to inhibit CK signaling in resistance against $H$. arabidopsidis, which may form a feedback mechanism to fine-tune the effect of CK in plant immunity (Argueso et al. 2012).
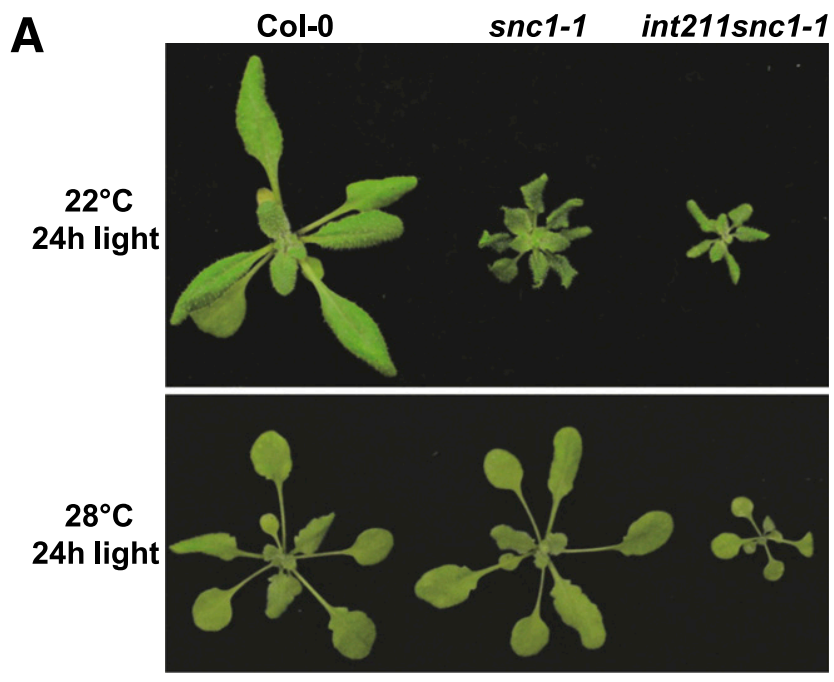

B

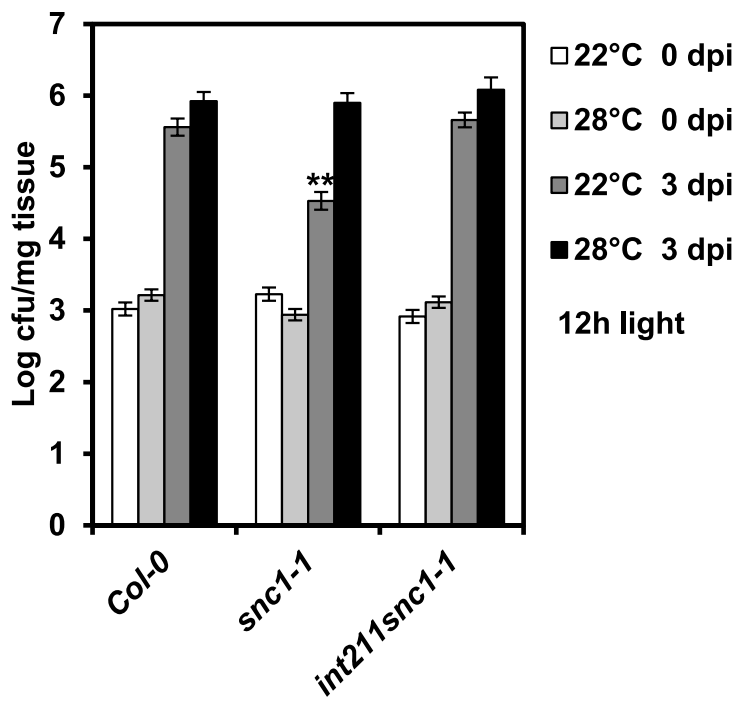

Fig. 1. The int211 snc1-1 double mutant has enhanced dwarf phenotype at 22 and $28^{\circ} \mathrm{C}$. A, Growth phenotypes of wild-type Col-0, snc1-1, and int 211 snc $1-1$ grown at 22 and $28^{\circ} \mathrm{C}$ under constant light at 14 days after germination. B, Growth of Pseudomonas syringae pv. tomato DC3000 in Col-0, snc1-1, and int 211 sncl-1 plants grown under $12 \mathrm{~h}$ of light at 22 and $28^{\circ} \mathrm{C}$ at 0 and 3 days postinoculation from three sets of seedlings for each genotype and temperature combination. Values represent means \pm standard deviation $(n=3)$. Asterisks $(* *)$ indicate a significant difference from the wild-type plants $(P<0.05$ according to a Student's $t$ test). Similar results were obtained in three independent experiments.
Here, we report a role of a $t$ Z-type CK transporter gene, $A t A B C G 14$, in plant immunity, through a genetic screen for modifiers of autoimmune mutant sncl-1. We found that the loss of AtABCG14 suppresses snc1-1 autoimmunity and downregulates the expression of $S N C 1$. In addition, exogenous application of $t Z$-type $C K$ enhances basal resistance to $P$. syringae pv. tomato DC3000 and enhances expression of SNC1. Together, the results in this study revealed an important role of tZ-type CK in plant disease resistance.

\section{RESULTS}

The int211 mutation suppresses snc1-1 autoimmunity.

The snc1-1 mutant has a dwarf phenotype at $22^{\circ} \mathrm{C}$, resulting from constitutive defense responses, but it is wild type in appearance at $28^{\circ} \mathrm{C}$, due to high temperature suppression of defense responses (Yang and Hua 2004). A genetic screen for mutants with dwarf phenotype in the sncl-1 background at $28^{\circ} \mathrm{C}$ was carried out to identify int mutants that have enhanced resistance at high temperature (Zhu et al. 2010). One putative mutant, int 211snc1-1, displayed a similar dwarf phenotype at 22 and $28^{\circ} \mathrm{C}$ (Fig. 1A). However, unlike several other int sncl mutants in which the growth defect is correlated with enhanced disease resistance (Mang et al. 2012; Zhu et al. 2010, 2012), this int 211snc1-1 mutant did not have an increased disease resistance at $28^{\circ} \mathrm{C}$. These plants were inoculated with virulent pathogen $P$. syringae pv. tomato DC3000 and pathogen growth was measured at day 0 and day 3 after inoculation (Fig. 1B). At $22^{\circ} \mathrm{C}$, the snc1-1 plants showed enhanced resistance to the pathogen, while int 211sncl-1 had much reduced resistance compared with sncl-1 and supported similar levels of pathogen growth as the wild type (Fig. 1B). At $28^{\circ} \mathrm{C}$, all the plants supported the same amount of bacterial growth at 3 days after inoculation (Fig. 1B). Therefore, a mutation in the int211snc1-1 plant or, more likely, the same mutation causing the dwarf phenotype of int 211 is also a suppressor of the enhanced disease resistance phenotype of sncl-1.

\section{Cloning of the INT211 gene as ABCG14.}

To identify the causal mutation for the growth and defense defects in int211snc1-1, we performed next-generation sequencing for mapping and gene identification (Zhu et al. 2012). The int211sncl-1 double mutant was crossed to the original snc1-1 mutant, and the int211snc1-1 double mutant-looking plants segregated at one out of four in the F2 progenies grown at $28^{\circ} \mathrm{C}$. Approximately 100 int 211 sncl-1-looking plants and 200 snc 1-1-looking plants were collected and pooled. Genomic DNAs were extracted and purified from these mutant and nonmutant pools, and libraries constructed from these DNAs were subject to next-generation sequencing on the Illumina Hi-Seq 2500 machine with single-end reads of $100 \mathrm{bp}$.

We obtained approximately $25 \times$ coverage of the Arabidopsis genome for each of the two samples. By searching for enrichment of mutant alleles in the mutant pool (Zhu et al. 2012), we identified one major peak on chromosome I. Seven consecutive single nucleotide polymorphisms (SNPs) between positions $10,928,363$ and $12,450,361$ were found to have nonreference alleles in all reads from the mutant pool but not those from the nonmutant pool, indicating that they are int 211 candidate mutations. On the basis of annotation by ANNOVAR (Wang et al. 2010), two of seven SNPs were alterations in the intergenic or noncoding regions and five caused nonsynonymous mutations (Fig. 2A). We subsequently obtained two T-DNA insertion lines (Alonso et al. 2003) for each of the seven candidate genes from the Arabidopsis Biological Resource Center. Homozygous mutants were isolated for these 14 lines and only SK_15917 (abcg14-1) and SK_15918 (abcg14-2), both in ABCG14 (At1g31770), exhibited a dwarf phenotype. We suspect that this 
could be the single mutant phenotype of int 211 , because a similar phenotype segregated in the $\mathrm{F} 2$ population of a cross between int211snc1-1 and Col-0. The int 211 snc1-1 contains a transition mutation of $\mathrm{C}$ to $\mathrm{T}$, which results in substitution of serine with phenylalanine in the ABCG14 protein (Fig. 2A).

To verify that this mutation in $A B C G 14$ is indeed the causal mutation of the growth defect of int211snc1-1, we crossed snc1-1 with two ABCG14 mutant alleles, abcg14-1 and abcg14-2, respectively, and isolated the abcg14-1snc1-1 and abcg14-2snc1-1 double mutants. The double mutants exhibited a dwarf phenotype similar to int 211 snc $1-1$ at both 22 and $28^{\circ} \mathrm{C}$ (Fig. 2B and $\mathrm{C}$ ). In addition, we introduced a functional enhanced green fluorescent protein $(e G F P)$ and $A B C G 14$ fusion gene $p A B C G 14:: E G F P-A B C G 14$ to int 211 sncl-1 by crossing (Zhang et al. 2014). The transgenic plants of $p A B C G 14:$ : EGFP-ABCG14 in int211 sncl-1 exhibited a snc1-1-like phenotype at both $22^{\circ} \mathrm{C}$ and $28^{\circ} \mathrm{C}$ (Fig. $2 \mathrm{~B}$ and C), indicating that $A B C G 14$ is, indeed, the INT211 gene for the growth phenotype. We therefore referenced int211 as abcg14-3.

Reduced growth and defense in abcg14snc1-1 both result from the loss of $A B C G 14$ function.

The abcgl4 mutants had retarded shoot growth, smaller rosette leaves, and thinner stems. Because cytokine has been shown to promote cell division, we analyzed the expression of some core cell-cycle regulating genes, including CDT1A, MCM2, CYCD3;1, CDC20.1, and CYCA2;1 (Menges et al. 2005). $C D T 1$ and $M C M 2$ are predominantly expressed in $\mathrm{G} 1 / \mathrm{S}$ transition and early $\mathrm{S}$ phase; $C Y C D 3 ; 1$ is in both $\mathrm{G} 1 / \mathrm{S}$ and G2/M transitions; CDC20.1 is in the $\mathrm{S}$ and $\mathrm{M}$ phase; and CYCA2; 1 is in the G2/M transition. The abcgl4 mutant had increased expression of $C D T 1 A$ and $M C M 2$ genes and reduced

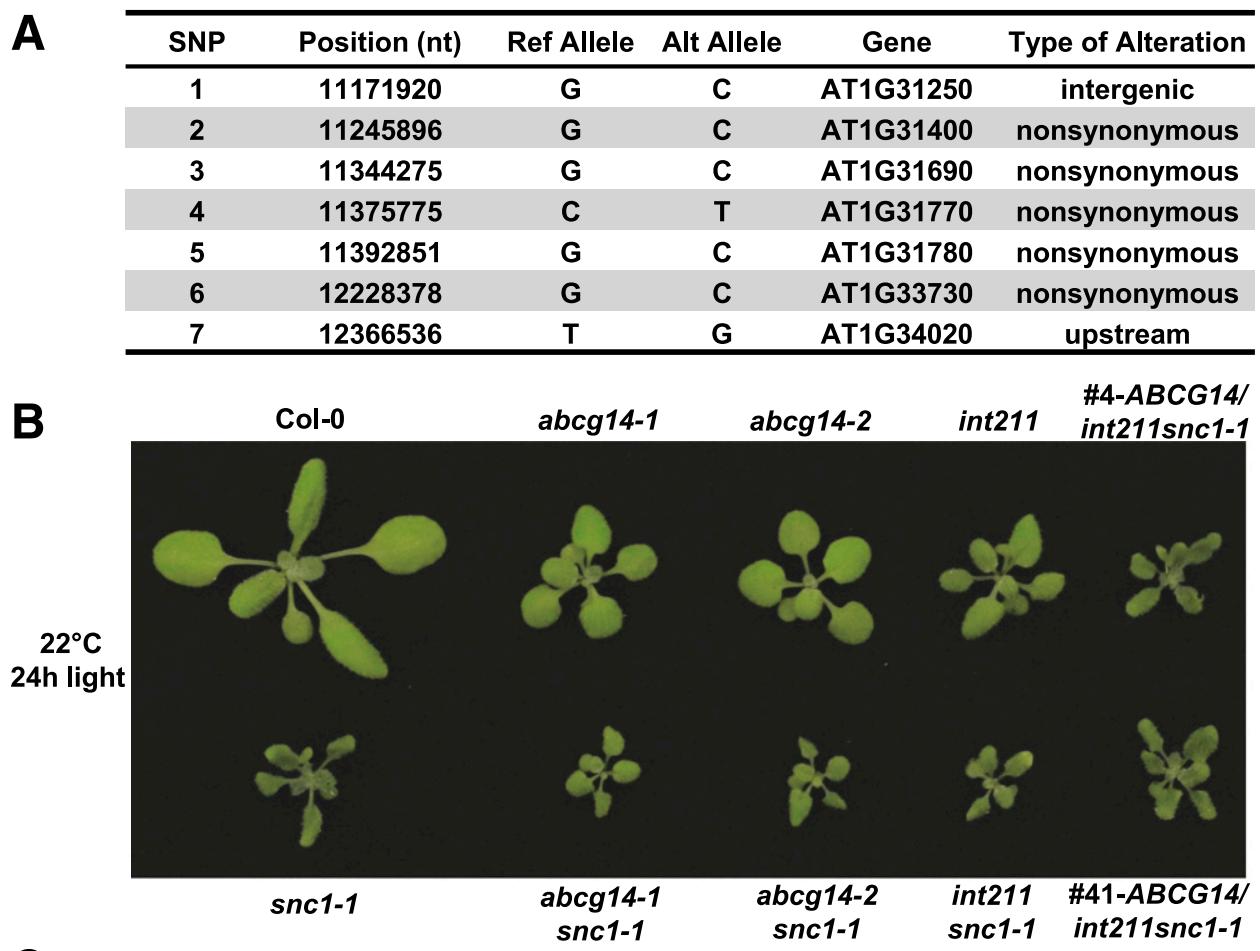

C

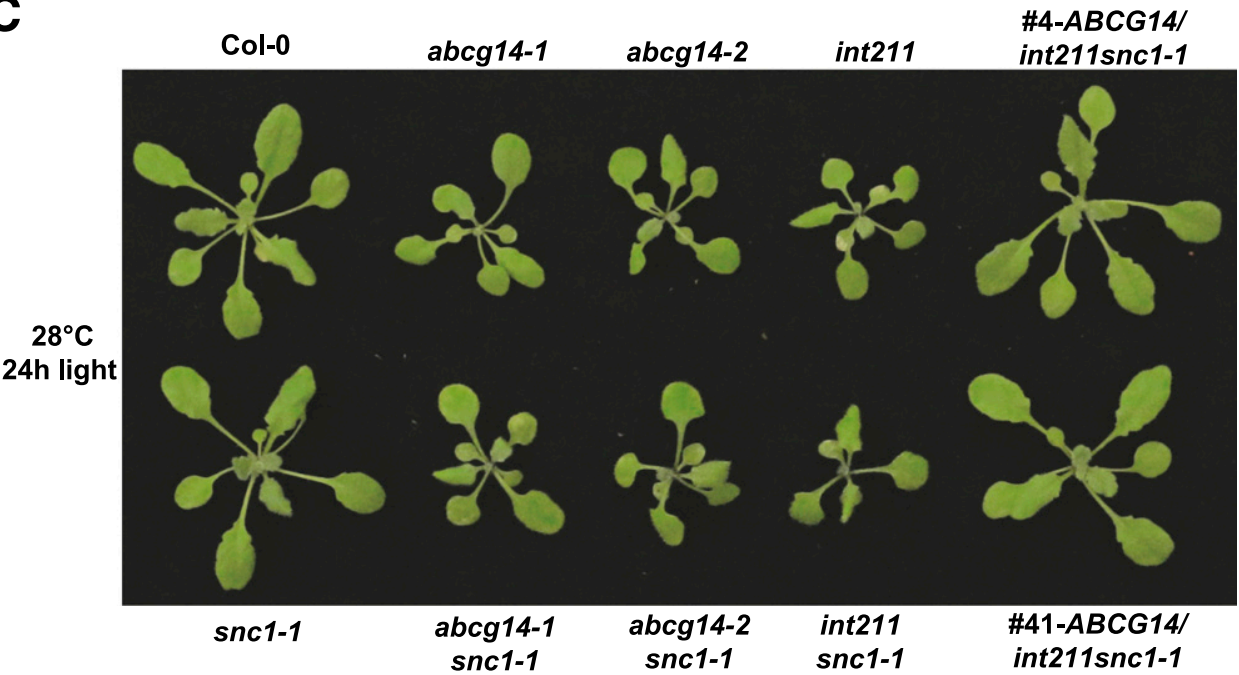

Fig. 2. Identification of the int 211 mutation. A, Annotation of all seven candidate single nucleotide polymorphisms (SNPs) in the defined int 211 region. The base position, sequence of the reference allele, sequence of the nonreference allele, its associated gene, and type of alteration for each SNP are shown. $\mathbf{B}$ and $\mathbf{C}$, Phenotype of abcg14-1 and abcg14-2 single mutants, abcg $14 \operatorname{snc} 1-1$ double mutants, and complementation lines in int 211 sncl-1 grown at $22^{\circ} \mathrm{C}$ (B) and $28^{\circ} \mathrm{C}$ (C) under constant light for two weeks. 
expression of the $C Y C D 3 ; 1, C D C 20.1$, and $C Y C A 2 ; 1$ genes (Fig. 3A). This suggests that mitosis is reduced in the mutant, which accounts, at least partially, for the growth defect.

We subsequently quantified the biomasses as a readout of growth, to determine how $a b c g 14$ and sncl-1 interact to impact the growth. The wild type, snc1-1, abcg14-3, and abcgl43 snc1-1 plants were grown under constant light for 14 days after germination, before measurement. At $22^{\circ} \mathrm{C}$, the sncl-1 and $a b c g 14-3$ single mutants were 48 and $30 \%$ of the wild-type weight and the double mutant was $14 \%$ of the wild-type weight (Fig. 3B). At $28^{\circ} \mathrm{C}$, sncl-1 had a similar weight to the wild type, due to a lack of defense activation, and the abcg14-3 and abcg14-3snc1-1 mutants were both approximately $28 \%$ of the wild-type weight (Fig. 3B). These results indicate that abcgl4
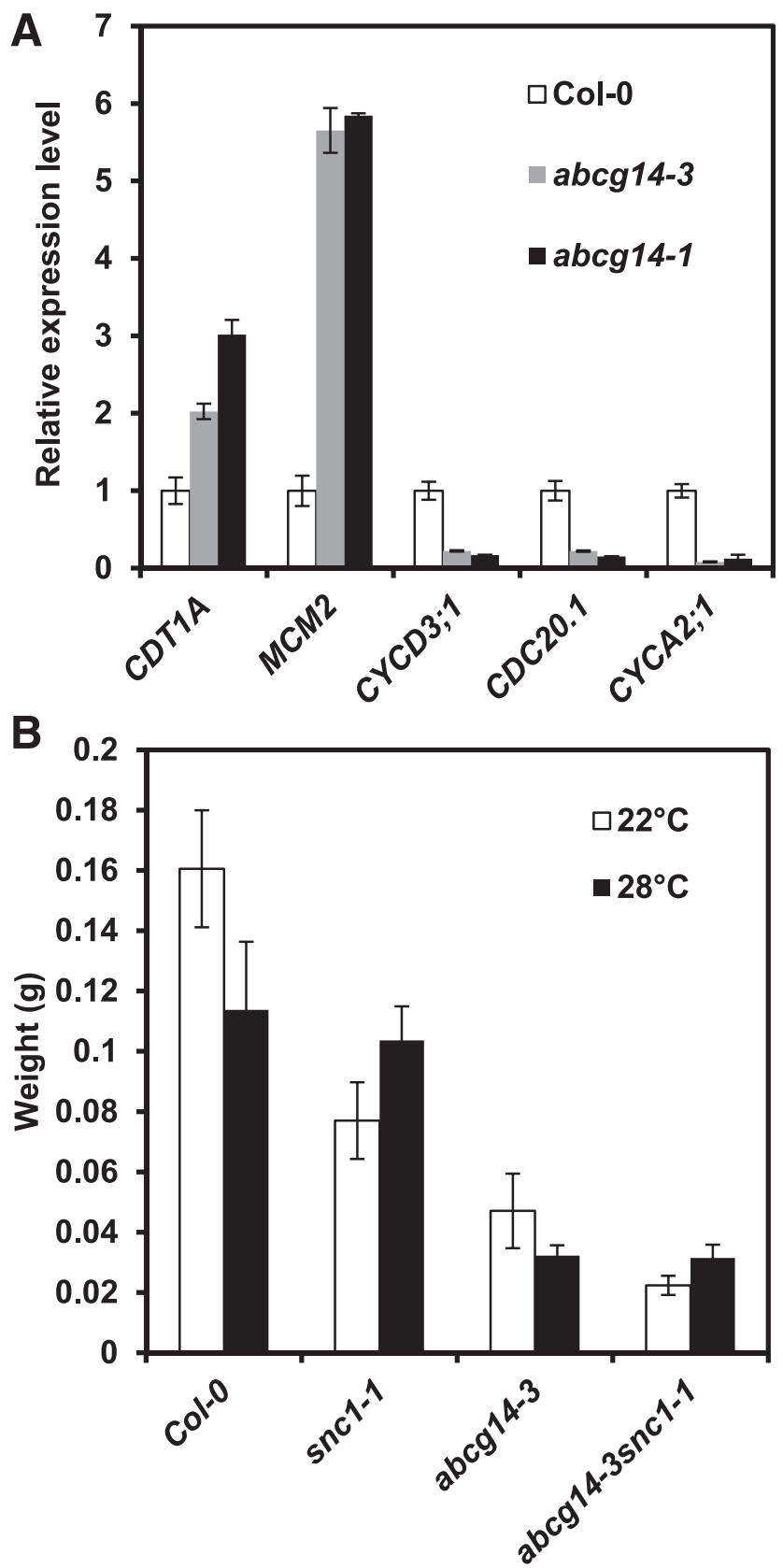

Fig. 3. $A B C G 14$ mutations have an additive effect on snc1-1. A, The expression of cell cycle-related genes in abcg 14 mutants and wild type. B, Fresh weight of Col-0, abcg14-3, snc1-1, and abcg14-3snc1-1 mutants grown at 22 and $28^{\circ} \mathrm{C}$ under constant light for 2 weeks after germination (mean value \pm standard deviation $[n=20]$ ). has an additive interaction with sncl-1 to affect growth at both 22 and $28^{\circ} \mathrm{C}$.

We further asked whether or not the mutation in $A B C G 14$ is also responsible for the suppressed disease resistance in int 211 sncl-1 compared with sncl-1. To this end, we analyzed bacterial growth of $P$. syringae pv. tomato DC3000 in the double mutants sncl-1 with two abcg14 alleles. Both the abcg14-1 and the abcg14-2 mutations suppressed the disease resistance phenotype of sncl-1 at $22^{\circ} \mathrm{C}$ (Fig. 4A). In addition, the transgene pABCG14::EGFP$A B C G 14$ that rescued the growth defect of int211snc1-1 also rescued its defense phenotype. The transgenic line $p A B C G 14:$ : EGFP-ABCG14 int211snc1-1 showed enhanced resistance to pathogen $P$. syringae pv. tomato DC3000 when compared with int211snc1-1, to a similar resistance level as that of sncl-1 (Fig. 4A). Consistent with the disease resistance phenotype, accumulation of $S N C l$ and PRI transcripts is also affected by $A B C G 14$. Analysis by quantitative reverse transcription-polymerase chain reaction (PCR) revealed that both $S N C l$ and $P R 1$ genes had a higher expression level in the sncl-1 single mutant and were reduced to the wild-type levels in the abcg14-3sncl-1 and abcg141snc1-1 double mutants (Fig. 4B and C). Therefore, ABCG14 is the INT211 gene and it is a positive regulator for both plant growth and SNC1-mediated disease resistance.

\section{Exogenous application of $t \mathrm{Z}$-type $\mathrm{CK}$ rescues growth defects of abcg14 but not abcg14snc1-1.}

$A B C G 14$ was shown to mediate the translocation of $t$ Z-type $\mathrm{CK}$ from roots to shoots via xylem, to regulate shoot growth (Ko et al. 2014; Zhang et al. 2014). We hypothesized that the enhanced growth defect of abcg 14-3sncl-1 compared with that of sncl-1 is due to a deficiency of $t$ Z-type CK. To test this, we applied iP and $t Z$ types of CK externally to the plants every day. Application of the $t Z$ type but not much of the iP type of CK induces a slight elongation of petioles in both the wild type and abcg 14-3 mutant at $22^{\circ} \mathrm{C}$ (Fig. 5A and C). Among the different concentrations tested, $10 \mu \mathrm{M}$ of $t \mathrm{Z}$-type CK had the best effect in rescuing the smaller rosette phenotype of the abcg14-3 single mutant at $22^{\circ} \mathrm{C}$, and the $a b c g 14-3$ mutant grew similarly to the wild type with this application (Fig. 5A and C). Surprisingly, CK application did not revert the abcg14-3snc1-1 double mutant phenotype to the sncl-1 single mutant phenotype, and the abcg14-3snc1-1 remained the same dwarf phenotype with or without $\mathrm{CK}$ application (Fig. $5 \mathrm{~A}$ and C). At $28^{\circ} \mathrm{C}$, the $t \mathrm{Z}$-type (not the iP-type) CK partially rescued the growth defects of the abcg14-3 and the abcg14-3sncl-1 mutants, and these two mutants grew larger with $t Z$-type $C K$ application than without application but were smaller than wild type or sncl-1 without CK application (Fig. 5B and C).

The apparent lack of effect of CK application on abcg14-3 sncl-1 growth phenotype at $22^{\circ} \mathrm{C}$ prompted us to investigate the effect of CK application on defense responses independent of its promoting effect on plant growth in sncl-1. Indeed, the sncl-1 single mutant plants were smaller with the treatment of $t$ Z-type CK and their leaves had more pronounced watersoaked phenotype associated with defense activation at $22^{\circ} \mathrm{C}$ and constant light (Fig. 5A). The abcg14-3snc1-1 double mutant plants developed leaves that were more curved and water-soaked with $t$ Z-type CK application (Fig. 5A). We also analyzed plants grown under a 12-h light and 12-h dark photoperiod, in which defense activation in sncl-1 is milder than under constant light. Indeed, the abcg14-3sncl-1 double mutant has a milder growth defect grown under $12 \mathrm{~h}$ of light than under constant light, likely due to a more complete inhibition of defense activation from snc1-1 by the abcg14-3 mutation (Fig. 5D). Again, $t$ Z-type CK rescued the abcg14-3 single mutant to the size to the wild type, but it did not rescue the abcg14-3sncl-1 double mutant to the size of sncl (Fig. 5D). Furthermore, 
application of $t Z$-type CK enhanced the water-soaked and curveddown phenotype in leaves of sncl-1 and abcg14-3sncl-1 grown under $12 \mathrm{~h}$ of light, similarly to those seen under constant light (Fig. $5 \mathrm{D})$. These observations suggest that exogenous application of $t \mathrm{Z}$ type CK may enhance plant immune responses in sncl-1. We hypothesized that deficiency of $t$-type CK simultaneously reduces growth and suppresses immune responses in snc1-1, and application of $t Z$-type $\mathrm{CK}$ not only brings back but, also, further enhances immunity in abcg14-3snc1-1 leading to growth inhibition.

\section{Exogenous application of $t Z$-type CK enhances plant disease resistance.}

To further test the above hypothesis, we performed a pathogen growth assay after spraying with $\mathrm{CK}$ under the 12-h light and 12-h dark condition. The plants of Col-0, snc1-1, abcg14-3, and $a b c g 14-3$ sncl-1 were sprayed with CK daily for 10 days before inoculation with the virulent pathogen $P$. syringae pv. tomato DC3000 (Fig. 6A). The four concentrations (0.01, 0.1, 1 , and $10 \mu \mathrm{M})$ used gave similar results, and the $10-\mu \mathrm{M}$ data are presented here. The $t \mathrm{Z}$-type CK conferred a significant reduction of pathogen growth in all four genotypes compared with plants sprayed with iP-type CK or the solvent control. The enhancement of resistance was most drastic in the abcgl43 snc $1-1$ double mutant, with a more than 38-fold decrease in pathogen growth (Fig. 6B).

We subsequently analyzed the expression of defense-related genes with application of the $t$ Z-type CK. Daily spray with $t$ Ztype $\mathrm{CK}$ increased expression of $P R I$ by fourfold compared with the control. Interestingly, $S N C 1$ expression is also increased by about fourfold (Fig. 6C). This result indicates that application of $t \mathrm{Z}$-type $\mathrm{CK}$ enhances basal disease resistance and increases $S N C 1$ gene expression.

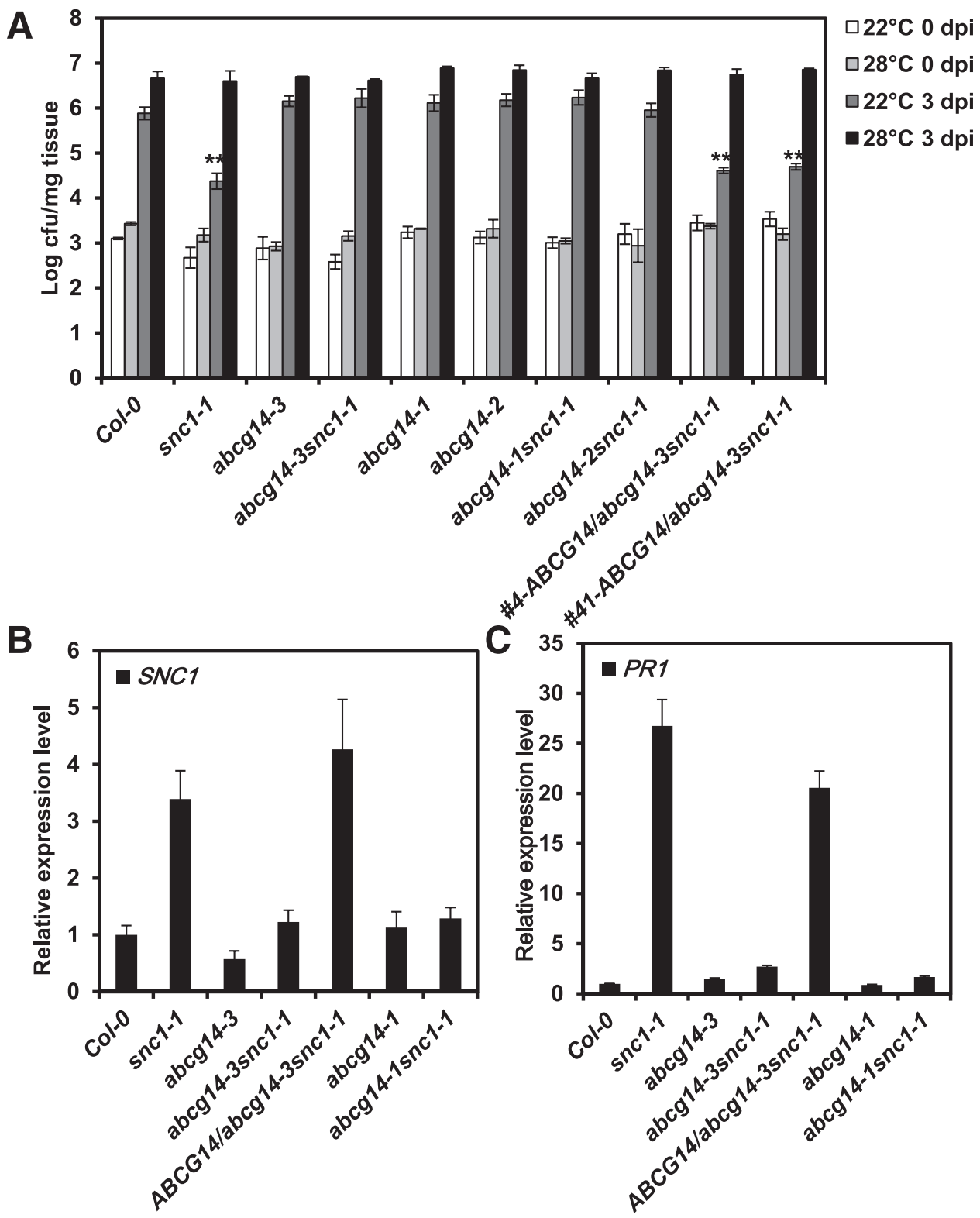

Fig. 4. Loss-of-function mutation in ABCG14 suppresses the constitutive defense response in snc1-1. A, Growth of Pseudomonas syringae pv. tomato DC3000 in Col-0, abcg 14, snc1-1, abcg14snc1-1, and complementation lines grown under a 12-h light condition at 22 and $28^{\circ} \mathrm{C}$, at 0 and 3 days postinoculation, from three sets of seedlings in each sample. Values represent means \pm standard deviation $(n=3)$. Similar results were obtained in three independent experiments. Asterisks $(* *)$ indicate a significant difference from wild-type plants $(P<0.05$ according to a Student's $t$ test). B, Expression of $S N C 1$ and $\mathbf{C}$, $P R 1$ in abovementioned plants grown under constant light for 2 weeks, as assayed by reverse transcription-polymerase chain reaction. Actin was used as internal control. Three biological repeats were performed and similar results were obtained. 


\section{DISCUSSION}

CKs act as both long-range and local signals to regulate cell division and differentiation throughout plant development, such as chloroplast development, leaf senescence, and root- and shoot apical dominance (Chory et al. 1994; Davies et al. 1966; Matsumoto-Kitano et al. 2008). Recently, a role of CKs in plant immunity has also been discovered and investigated. Exogenous application of $t$-type $\mathrm{CK}$ or overexpression of $\mathrm{CK}$ biosynthetic enzyme genes exhibited enhanced resistance to virulent bacterial pathogen $P$. syringae pv. tomato DC3000 in Arabidopsis (Choi et al. 2010; Naseem et al. 2012). In contrast, CK receptor mutant $a h k 2 a h k 3$ and transgenic line overexpression of CK-degrading enzyme genes such as $C K X 2$ and $C K X 4$ displayed enhanced susceptibility to $P$. syringae pv. tomato DC3000 (Choi et al. 2010). In this study, we found further evidence for the role of $t$-type $\mathrm{CK}$ in resistance against $P$. syringae pv. tomato DC3000 and revealed a new target of $\mathrm{CK}$ in plant immunity.

$\mathrm{ABCG} 14$ is an $\mathrm{ABC}$ transporter, shown to translocate the $t \mathrm{Z}$ type of CK from roots to shoots through xylem (Ko et al. 2014; Zhang et al. 2014). Plants without ABCG14 showed abnormal expression of key cell cycle-related genes, which likely accounts, at least partially, for the growth defect in the abcg14 mutant (Fig. 3A). The abcgl4 and sncl-1 mutations displayed an additive effect on growth inhibition in terms of biomass and plant size, indicating that $\mathrm{CK}$ and plant immune receptor $S \mathrm{NC1}$ mediated autoimmunity affect plant growth independently (Figs. $2 \mathrm{~B}$ and $\mathrm{C}$ and $3 \mathrm{~B}$ ). $\mathrm{CK}$ has numerous effects on plant growth, including the development of xylem and phloem cells in the vascular bundle (Zhang et al. 2014). It would be interesting to determine, in the future, how other aspects of growth besides biomass and size are affected by the combination of immune activation and $\mathrm{CK}$ pathway perturbation.

The $t Z$-type CK is required for defense responses mediated by $S N C l$, as autoimmunity of $s n c 1-1$ is suppressed by the deficiency of $t \mathrm{Z}$-type $\mathrm{CK}$ in the abcg14 mutation. Interestingly, a gain-offunction mutant of another immune receptor, UNI (uni-1D), showed higher accumulation of $t$ Z-type CK but not iP-type CK (Hwang et al. 2012; Igari et al. 2008). The rin4 knockdown mutant, in which the two immune receptor proteins RPS2 and RPM1 are activated, also had upregulation of CK ARR transcription factor genes (Igari et al. 2008). The CK pathway activation was postulated to mediate, at least partially, the growth effect from immunity activation. It is not yet determined whether either the $\mathrm{CK}$ amount, the CK response, or both are activated in the sncl-1 mutant. On the other hand, application of $t$ Z-type CK enhances disease resistance to pathogen $P$. syringae pv. tomato DC3000 in wild type and snc1-1, indicating that CK activation might contribute to defense activation in the autoimmune mutants and during defense responses against pathogens. CK (BA type) was shown to have a concentration-dependent effect on resistance to $H$. arabidopsidis (Argueso et al. 2012). We did not observe an obvious concentration-dependent effect of $t \mathrm{Z}$-type CK on resistance to $P$. syringae pv. tomato DC3000. Future study is needed to measure endogenous CK concentrations after exogenous application, to determine whether or not there is a difference in concentration effect of $\mathrm{CK}$ between resistance to the
A

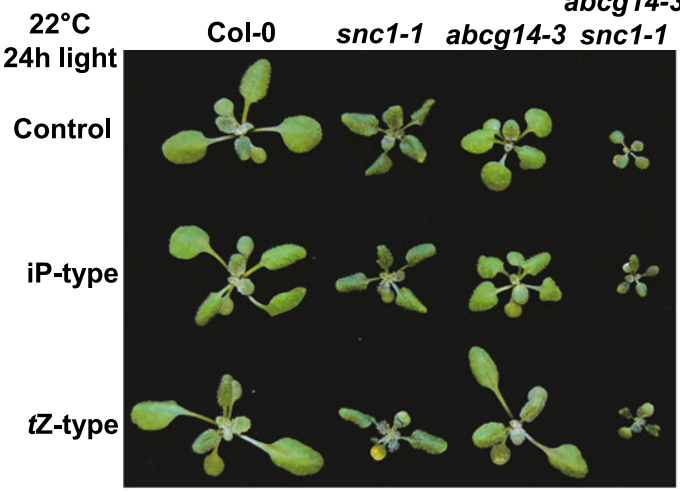

B

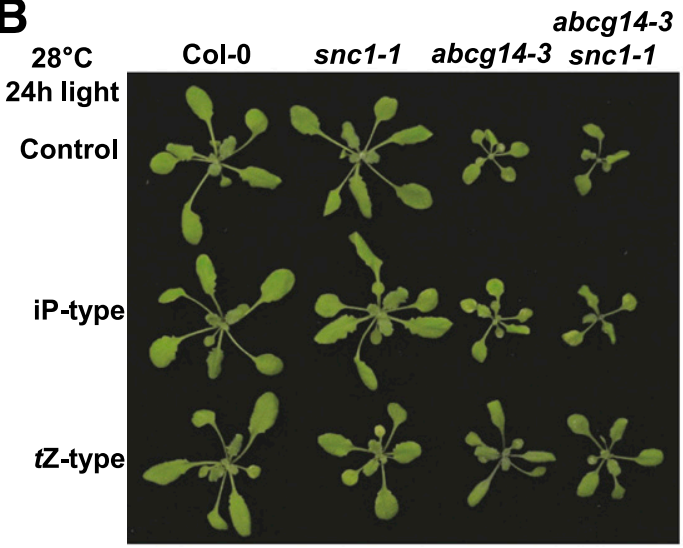

C

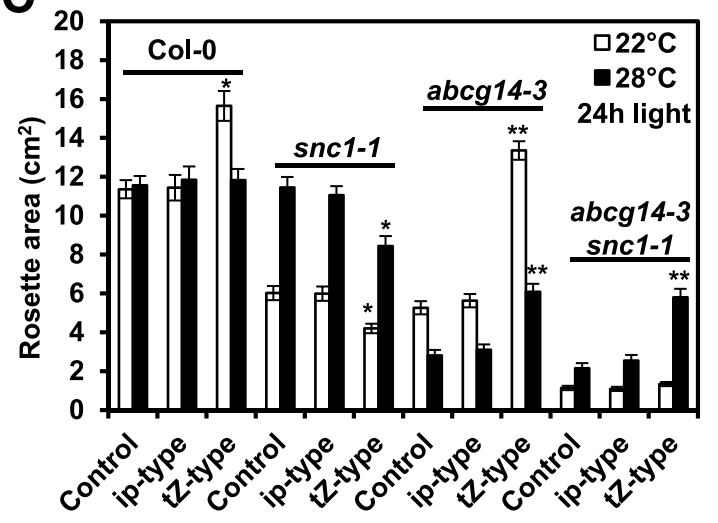

D

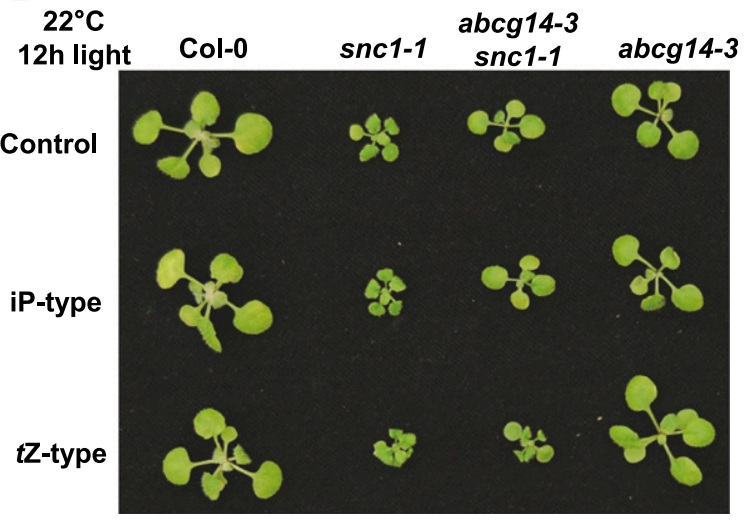

Fig. 5. Exogenous application of $t$ Z-type cytokinin rescues the $a b c g 14$ single but not the abcg14sncl-1 double mutant growth phenotype. A and B, Phenotype of wild type, snc1-1, abcg 14-3, and $a b c g 14-3 s n c 1-1$ plants grown under constant light and sprayed with $0.1 \%$ dimethyl sulfoxide (control) solution, $10 \mu \mathrm{M}$ iPtype, and $10 \mu \mathrm{M} t \mathrm{Z}$-type cytokinin once a day for 10 days, starting from 5 days after sowing at $22^{\circ} \mathrm{C}(\mathrm{A})$ and $28^{\circ} \mathrm{C}(\mathrm{B})$ respectively. $\mathbf{C}$, Quantification of rosette areas of plants in A and B. The area is calculated from the smallest circle encompassing the rosette. Values are mean \pm standard deviation $(n=10)$. Asterisks $(*)$ indicate a significant difference from each control plant $(P<0.05$ according to a Student's $t$ test). D, Phenotype of the same plants as in $(A)$ but grown under a 12-h light and 12-h dark photoperiod. 
hemibiotrophic P. syringae pv. tomato DC3000 and that to the biotrophic $H$. arabidopsidis pathogens.

Earlier studies found a synergistic effect of CK and SA in the plant immune signaling network and the effect was attributed to $\mathrm{CK}$ response transcription factors that also regulate plant immunity genes. An increase or a decrease of the positive CKregulating transcription factor ARR2 lead to enhanced or decreased resistance to bacterial pathogen $P$. syringae pv. tomato DC3000, respectively. ARR2 is shown to interact directly with the SA transcription factor TGA3, to positively regulate PRl gene expression (Choi et al. 2010, 2011). The negative CK-regulating type A ARR transcription factors are found to be negative regulators of resistance to biotrophic pathogen H. arabidopsidis Noco2 (Argueso et al. 2012). Overexpression of a CK response factor 2 (AtCRF2) leads to autoimmune response by enhancing SA biosynthesis in Arabidopsis (Kwon 2016). The direct binding targets of these transcription factors in the later cases were not known.

Here, we found a deficiency in $t Z$-type $C K$ led to the reduction of expression of $R$ gene $S N C 1$ (Fig. 4B) and exogenous application of $t$ Z-type CK upregulated $S N C l$ expression (Fig. 6C). Therefore, expression of plant immune receptor genes could be another target of CK intersection with plant immunity. It will be interesting to test whether or not CK-response

A
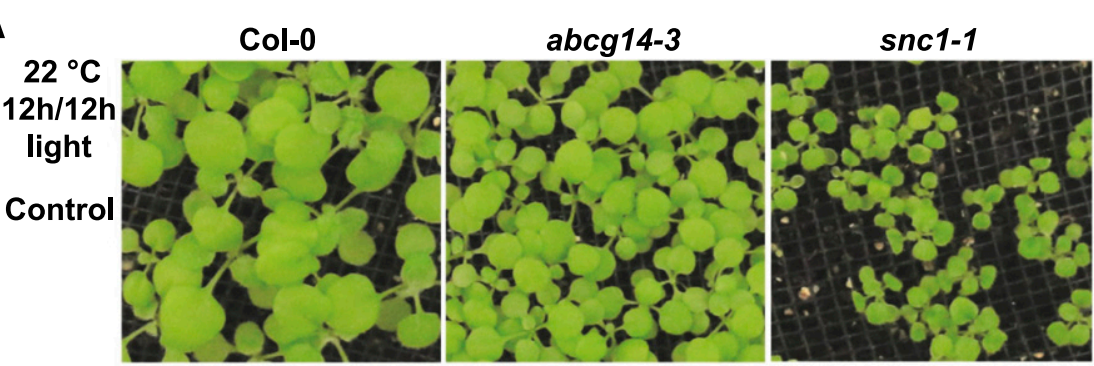

abcg14-3snc1-1
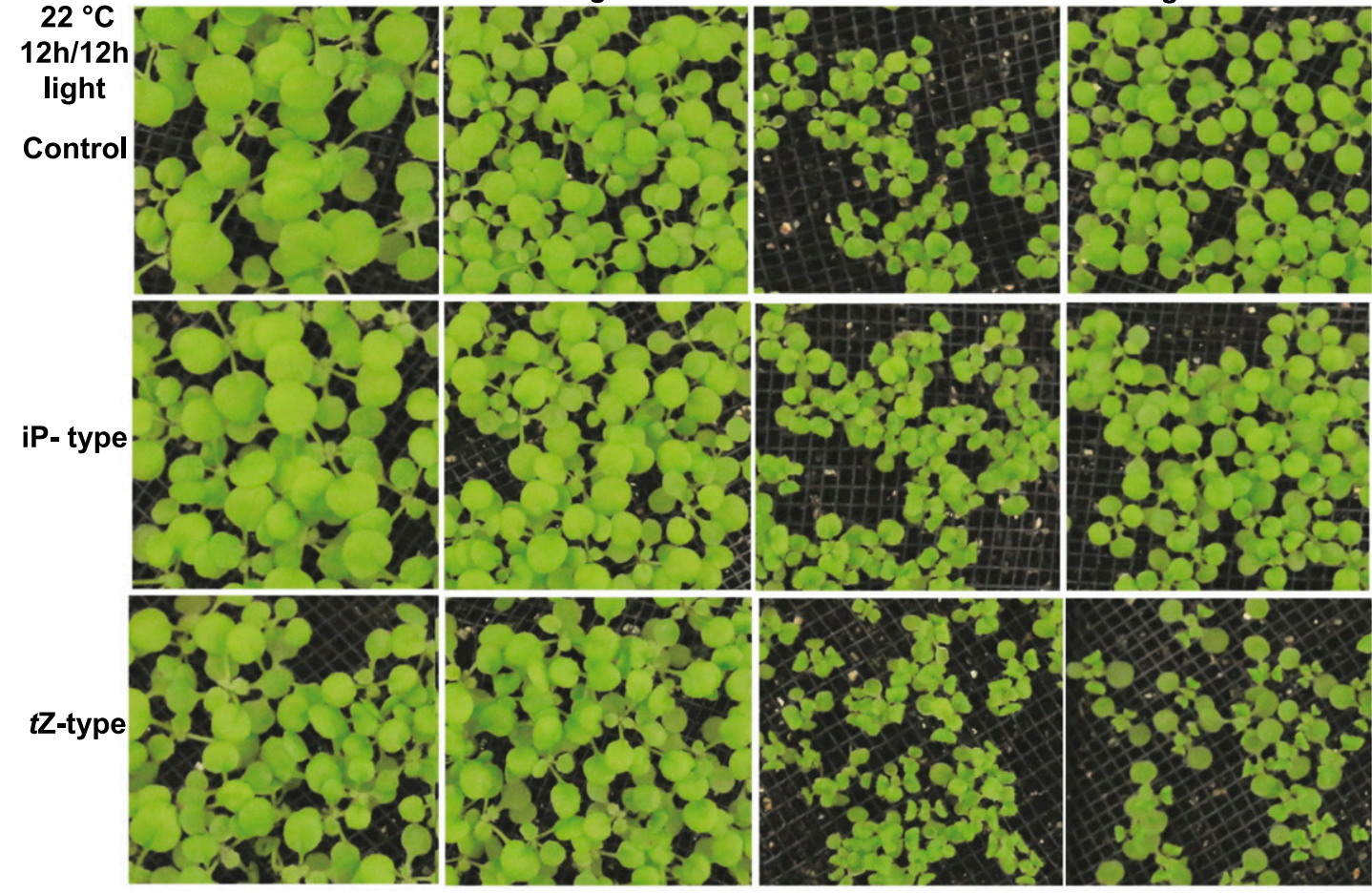

B
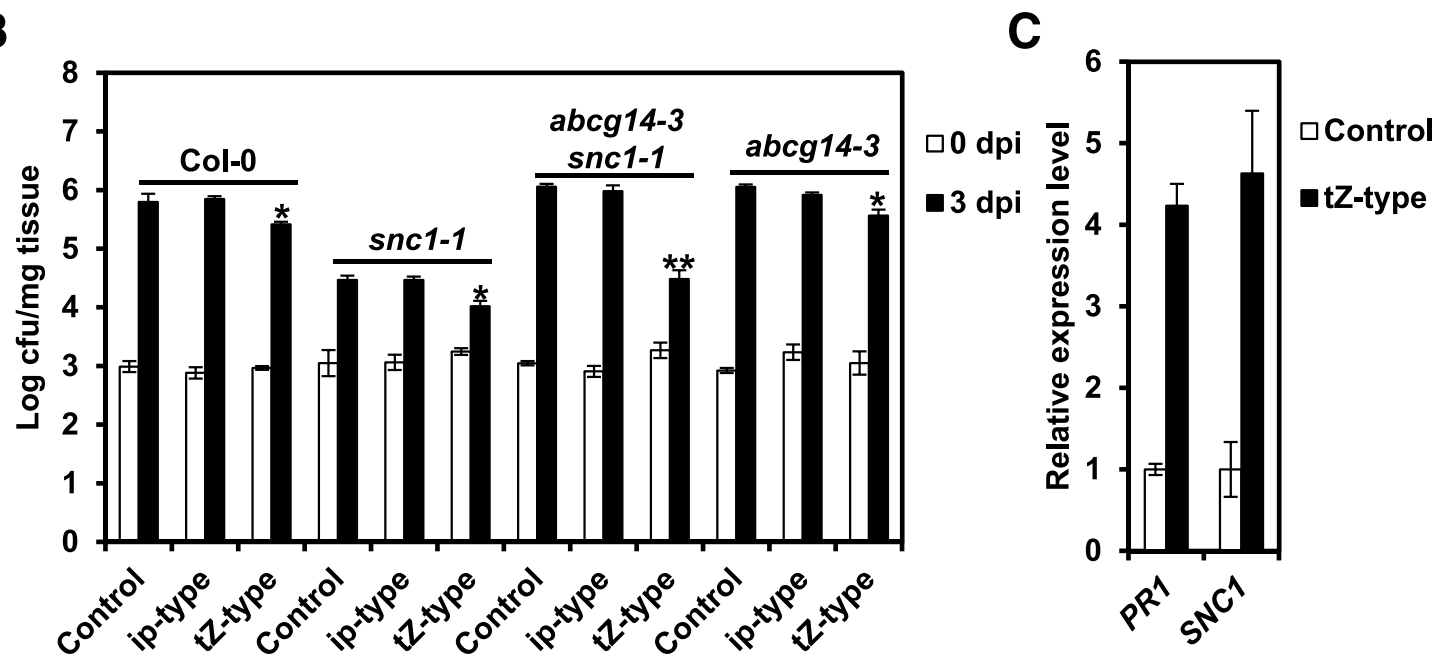

Fig. 6. Exogenous application of $t$ Z-type cytokinin enhances plant disease resistance. A, Phenotype of wild-type, snc1-1, abcg14-3, and abcg14-3snc1-1 plants grown at $22^{\circ} \mathrm{C}$ under a 12 -h light and 12 -h dark photoperiod. They were sprayed with control solution, $10 \mu \mathrm{M} \mathrm{iP}$-type, and $10 \mu \mathrm{M} t \mathrm{Z}$-type cytokinin once a day for 10 days, starting from 5 days after sowing. B, Growth of bacterial strains of Pseudomonas syringae pv. tomato DC3000 at 0 and 3 days postinoculation in plants in A. Each genotype had three biological repeats in each experiment. Values represent means \pm standard deviation $(n=3)$. An asterisk $(*)$ indicates a significant difference from each control plant at $P<0.05$ and two askterisks $(* *)$ indicate a difference at $P<0.005$, according to a Student's $t$ test. Similar results were obtained in three independent experiments. C, Expression of $S N C l$ and $P R I$ is up-regulated in Col-0 plants sprayed with $10 \mu \mathrm{M} t \mathrm{Z}$-type cytokinin once a day for 14 days, starting from 5 days after sowing and grown at $22^{\circ} \mathrm{C}$ under a 12 -h light photoperiod. Actin was used as internal control. Three biological repeats were performed and similar results were obtained. 
transcription factors directly regulate expression of plant immune receptor genes. Future study should reveal the impact of immune receptor activation and $\mathrm{CK}$ responses on each other in both plant growth and plant immunity.

\section{MATERIALS AND METHODS}

\section{Plant material and growth conditions.}

Arabidopsis thaliana plants were grown on soil at 22 and $28^{\circ} \mathrm{C}$, with light intensity at $100 \mu \mathrm{mol} \mathrm{m} \mathrm{m}^{-2} \mathrm{~s}^{-1}$ and relative humidity at 50 to $70 \%$. Plants were grown under constant light for morphological phenotypic and gene expression analysis unless stated otherwise. Plants were grown under the condition of $12 \mathrm{~h}$ of light and $12 \mathrm{~h}$ of darkness for pathogen growth tests.

The $p A B C G 14:: E G F P-A B C G 14$ transgenic line (Zhang et al. 2014) was crossed to int211 single and int211sncl-1 double mutants, respectively, for complementation.

\section{Mutant screen and next-generation sequencing.}

Mutant screen was carried out as described by Mang et al. (2012) and Zhu et al. (2013). The sncl-1 seeds were treated with $0.25 \%$ ethane methylsulfonate for $12 \mathrm{~h}$. Approximately 30,000 M2 plants (derived from 3,000 M1 plants) were screened at $28^{\circ} \mathrm{C}$ for int mutants with the $22^{\circ} \mathrm{C}$ sncl-1-like dwarf phenotype. For mapping, F2 progenies from a cross of int 211 sncl-1 and snc1-1 were grown and were scored at $28^{\circ} \mathrm{C}$. Equal amounts of leaf tissues were collected from each plant and tissues from mutant and nonmutant plants were pooled separately for genomic DNA extraction, using the E.Z.N.A. SP plant DNA midi kit (Omega Bio-tek, Inc.). DNA libraries for Hi-Seq were constructed using NEBNext Ultra II DNA library prep kit (New England Biolabs). The libraries were run on Illumina Hi-Seq with single reads of $100 \mathrm{bp}$.

\section{Pathogen resistance assay.}

Pseudomonas syringae pv. tomato DC3000 was grown 2 to 3 days on King's B medium with $50 \mathrm{mg}$ of kanamycin per liter and were resuspended at $10^{5} \mathrm{CFU} \mathrm{ml}^{-1}$ in a solution of $10 \mathrm{mM}$ $\mathrm{MgCl}_{2}$ and $0.02 \%$ Silwet L 77. Two-week-old seedlings (grown under $12 \mathrm{~h}$ of light and $12 \mathrm{~h}$ of dark) were dipinoculated with bacteria and were kept covered for $1 \mathrm{~h}$, before being slowly uncovered. The amount of bacteria in plants was analyzed at $1 \mathrm{~h}$ after dipping (day 0 ) and 3 days after dipping (day 3). The aerial parts of three inoculated seedlings were pooled per sample, and three samples were collected for each genotype and time point. Seedlings were ground in $1 \mathrm{ml}$ of $10 \mathrm{mM}$ of $\mathrm{MgCl}_{2}$, and serial dilutions of the ground tissue were used to determine the number of CFU per gram of fresh leaf tissue.

\section{Real-time quantitative PCR and gene expression.}

Total RNA was extracted with TRIzol reagent (Invitrogen) from leaves of 2- to 3-week-old plants, according to the manufacturer's protocol. To remove any contaminating DNA, the samples were treated with DNase (RNase-free DNase kit; Promega). cDNAs were synthesized from total RNA by using AffinityScript QPCR cDNA synthesis kit (Agilent Technologies). Real-time quantitative PCR was performed on the $B I O$ $R A D$ PCR system using iQ SYBR Green supermix (Bio-Rad). Primers used in this study are presented in Supplementary Table S1.

\section{Exogenous CK application.}

Seeds were directly sowed on the soil. After 5 days of growth, the plants were sprayed with $0.01,0.1,1$, and $10 \mu \mathrm{M}$ of tZ-type or iP-type CK or $0.1 \%$ dimethyl sulfoxide $+0.02 \%$ Silwet 77 solution (control) every day.

\section{ACKNOWLEDGMENTS}

We thank the Arabidopsis Biological Resource Center for the Arabidopsis mutants and C. Liu for the pABCG14::EGFP-ABCG14 transgenic plant. This work was supported by grants from the National Science Foundation (IOS-0919914 and IOS-1353738) to J. Hua. S. Wang was supported by a fellowship from the China Scholarship Council. J. Hua and Shuai Wang designed the experiments; Shuai Wang, Shu Wang, L. Yang, and Y. Zhu performed experiments; Shuai Wang, Q. Sun, and J. Hua analyzed data; Y. Yuan provided supervison; and Shuai Wang and J. Hua wrote the paper.

\section{LITERATURE CITED}

Achard, P., Renou, J.P., Berthome, R., Harberd, N.P., and Genschik, P. 2008. Plant DELLAs restrain growth and promote survival of adversity by reducing the levels of reactive oxygen species. Curr. Biol. 18: 656-660.

Alonso, J. M., Stepanova, A. N., Leisse, T. J., Kim, C. J., Chen, H., Shinn, P., Stevenson, D. K., Zimmerman, J., Barajas, P., Cheuk, R., Gadrinab, C., Heller, C., Jeske, A., Koesema, E., Meyers, C. C., Parker, H., Prednis, L., Ansari, Y., Choy, N., Deen, H., Geralt, M., Hazari, N., Hom, E., Karnes, M., Mulholland, C., Ndubaku, R., Schmidt, I., Guzman, P., Aguilar-Henonin, L., Schmid, M., Weigel, D., Carter, D. E., Marchand, T., Risseeuw, E., Brogden, D., Zeko, A., Crosby, W. L., Berry, C. C., and Ecker, J. R. 2003. Genome-wide insertional mutagenesis of Arabidopsis thaliana. Science 301:653-657.

Argueso, C. T., Ferreira, F. J., Epple, P., To, J. P., Hutchison, C. E., Schaller, G. E., Dangl, J. L., and Kieber, J. J. 2012. Two-component elements mediate interactions between cytokinin and salicylic acid in plant immunity. PLoS Genet. 8:e1002448.

Bari, R., and Jones, J. D. 2009. Role of plant hormones in plant defence responses. Plant Mol. Biol. 69:473-488.

Browse, J. 2009. Jasmonate passes muster: A receptor and targets for the defense hormone. Annu. Rev. Plant Biol. 60:183-205.

Cao, F. Y., Yoshioka, K., and Desveaux, D. 2011. The roles of ABA in plant-pathogen interactions. J. Plant Res. 124:489-499.

Choi, J., Choi, D., Lee, S., Ryu, C. M., and Hwang, I. 2011. Cytokinins and plant immunity: Old foes or new friends? Trends Plant Sci. 16:388-394.

Choi, J., Huh, S. U., Kojima, M., Sakakibara, H., Paek, K. H., and Hwang, I. 2010. The cytokinin-activated transcription factor ARR2 promotes plant immunity via TGA3/NPR1-dependent salicylic acid signaling in Arabidopsis. Dev. Cell 19:284-295.

Chory, J., Reinecke, D., Sim, S., Washburn, T., and Brenner, M. 1994. A role for cytokinins in de-etiolation in Arabidopsis (det mutants have an altered response to cytokinins). Plant Physiol. 104:339-347.

Davies, C. R., Seth, A. K., and Wareing, P. F. 1966. Auxin and kinetin interaction in apical dominance. Science 151:468-469.

de Torres Zabala, M., Bennett, M.H., Truman, W.H., and Grant, M.R. 2009. Antagonism between salicylic and abscisic acid reflects early hostpathogen conflict and moulds plant defence responses. Plant J. 59: 375-386.

Derksen, H., Rampitsch, C., and Daayf, F. 2013. Signaling cross-talk in plant disease resistance. Plant Sci. 207:79-87.

Grant, M. R., and Jones, J. D. 2009. Hormone (dis)harmony moulds plant health and disease. Science 324:750-752.

Hammond-Kosack, K. E., and Jones, J. D. 1996. Resistance gene-dependent plant defense responses. Plant Cell 8:1773-1791.

Hann, D. R., Domínguez-Ferreras, A., Motyka, V., Dobrev, P. I., Schornack, S., Jehle, A., Felix, G., Chinchilla, D., Rathjen, J. P., and Boller, T. 2014. The Pseudomonas type III effector HopQ1 activates cytokinin signaling and interferes with plant innate immunity. New Phytol. 201:585-598.

Howe, G. A., and Jander, G. 2008. Plant immunity to insect herbivores. Annu. Rev. Plant Biol. 59:41-66.

Hwang, I., Sheen, J., and Müller, B. 2012. Cytokinin signaling networks. Annu. Rev. Plant Biol. 63:353-380.

Igari, K., Endo, S., Hibara, K., Aida, M., Sakakibara, H., Kawasaki, T., and Tasaka, M. 2008. Constitutive activation of a CC-NB-LRR protein alters morphogenesis through the cytokinin pathway in Arabidopsis. Plant J. 55:14-27.

Jiang, C. J., Shimono, M., Sugano, S., Kojima, M., Yazawa, K., Yoshida, R., Inoue, H., Hayashi, N., Sakakibara, H., and Takatsuji, H. 2010. Abscisic acid interacts antagonistically with salicylic acid signaling pathway in rice-Magnaporthe grisea interaction. Mol. Plant-Microbe Interact. 23: 791-798.

Jones, J. D., and Dangl, J. L. 2006. The plant immune system. Nature 444: 323-329. 
Katagiri, F., and Tsuda, K. 2010. Understanding the plant immune system. Mol. Plant-Microbe Interact. 23:1531-1536.

Kazan, K., and Manners, J. M. 2009. Linking development to defense: Auxin in plant-pathogen interactions. Trends Plant Sci. 14:373-382.

Ko, D., Kang, J., Kiba, T., Park, J., Kojima, M., Do, J., Kim, K. Y., Kwon, M., Endler, A., Song, W. Y., Martinoia, E., Sakakibara, H., and Lee, Y. 2014. Arabidopsis ABCG14 is essential for the root-toshoot translocation of cytokinin. Proc. Natl. Acad. Sci. U.S.A. 111: 7150-7155.

Kwon, T. 2016. Cytokinin response factor 2 positively regulates salicylic acid-mediated plant immunity in Arabidopsis thaliana. Plant Biotechnol. 33:207-210.

Mang, H. G., Qian, W., Zhu, Y., Qian, J., Kang, H. G., Klessig, D. F., and Hua, J. 2012. Abscisic acid deficiency antagonizes high-temperature inhibition of disease resistance through enhancing nuclear accumulation of resistance proteins SNC1 and RPS4 in Arabidopsis. Plant Cell 24: 1271-1284.

Matsumoto-Kitano, M., Kusumoto, T., Tarkowski, P., Kinoshita-Tsujimura, K., Václavíková, K., Miyawaki, K., and Kakimoto, T. 2008. Cytokinins are central regulators of cambial activity. Proc. Natl. Acad. Sci. U.S.A. 105:20027-20031.

Menges, M., de Jager, S. M., Gruissem, W., and Murray, J. A. H. 2005. Global analysis of the core cell cycle regulators of Arabidopsis identifies novel genes, reveals multiple and highly specific profiles of expression and provides a coherent model for plant cell cycle control. Plant J. 41: 546-566.

Nakashita, H., Yasuda, M., Nitta, T., Asami, T., Fujioka, S., Arai, Y., Sekimata, K., Takatsuto, S., Yamaguchi, I., and Yoshida, S. 2003. Brassinosteroid functions in a broad range of disease resistance in tobacco and rice. Plant J. 33:887-898.

Naseem, M., Philippi, N., Hussain, A., Wangorsch, G., Ahmed, N., and Dandekar, T. 2012. Integrated systems view on networking by hormones in Arabidopsis immunity reveals multiple crosstalk for cytokinin. Plant Cell 24:1793-1814.

Naseem, M., Wölfling, M., and Dandekar, T. 2014. Cytokinins for immunity beyond growth, galls and green islands. Trends Plant Sci. 19:481-484.

Navarro, L., Bari, R., Achard, P., Lison, P., Nemri, A., Harberd, N.P., and Jones, J.D. 2008. DELLAs control plant immune responses by modulating the balance of jasmonic acid and salicylic acid signaling. Curr. Biol. 18:650-655.

Navarro, L., Dunoyer, P., Jay, F., Arnold, B., Dharmasiri, N., Estelle, M., Voinnet, O., and Jones, J. D. 2006. A plant miRNA contributes to antibacterial resistance by repressing auxin signaling. Science 312: 436-439.
Pieterse, C. M., Leon-Reyes, A., Van der Ent, S., and Van Wees, S. C. 2009. Networking by small-molecule hormones in plant immunity. Nat. Chem. Biol. 5:308-316.

Siemens, J., Keller, I., Sarx, J., Kunz, S., Schuller, A., Nagel, W., Schmülling, T., Parniske, M., and Ludwig-Müller, J. 2006. Transcriptome analysis of Arabidopsis clubroots indicate a key role for cytokinins in disease development. Mol. Plant-Microbe Interact. 19: 480-494.

Ton, J., Flors, V., and Mauch-Mani, B. 2009. The multifaceted role of ABA in disease resistance. Trends Plant Sci. 14:310-317.

van Loon, L. C., Geraats, B. P., and Linthorst, H. J. 2006. Ethylene as a modulator of disease resistance in plants. Trends Plant Sci. 11:184-191.

Vlot, A. C., Dempsey, D. A., and Klessig, D. F. 2009. Salicylic acid, a multifaceted hormone to combat disease. Annu. Rev. Phytopathol. 47: 177-206.

Walters, D. R., and McRoberts, N. 2006. Plants and biotrophs: A pivotal role for cytokinins? Trends Plant Sci. 11:581-586.

Walters, D. R., McRoberts, N., and Fitt, B. D. 2008. Are green islands red herrings? Significance of green islands in plant interactions with pathogens and pests. Biol. Rev. Camb. Philos. Soc. 83:79-102.

Wang, K., Li, M., and Hakonarson, H. 2010. ANNOVAR: Functiona annotation of genetic variants from high-throughput sequencing data. Nucleic Acids Res. 38:e164.

Yang, S., and Hua, J. 2004. A haplotype-specific resistance gene regulated by BONZAI1 mediates temperature-dependent growth control in Arabidopsis. Plant Cell 16:1060-1071.

Yasuda, M., Ishikawa, A., Jikumaru, Y., Seki, M., Umezawa, T., Asami, T., Maruyama-Nakashita, A., Kudo, T., Shinozaki, K., Yoshida, S., and Nakashita, H. 2008. Antagonistic interaction between systemic acquired resistance and the abscisic acid-mediated abiotic stress response in Arabidopsis. Plant Cell 20:1678-1692.

Zhang, K., Novak, O., Wei, Z., Gou, M., Zhang, X., Yu, Y., Yang, H., Cai, Y., Strnad, M., and Liu, C. J. 2014. Arabidopsis ABCG14 protein controls the acropetal translocation of root-synthesized cytokinins. Nat. Commun. 5:3274.

Zhao, Y. 2008. The role of local biosynthesis of auxin and cytokinin in plant development. Curr. Opin. Plant Biol. 11:16-22.

Zhu, Y., Du, B., Qian, J., Zou, B., and Hua, J. 2013. Disease resistance geneinduced growth inhibition is enhanced by $r c d 1$ independent of defense activation in Arabidopsis. Plant Physiol. 161:2005-2013.

Zhu, Y., Mang, H. G., Sun, Q., Qian, J., Hipps, A., and Hua, J. 2012. Gene discovery using mutagen-induced polymorphisms and deep sequencing: Application to plant disease resistance. Genetics 192:139-146.

Zhu, Y., Qian, W., and Hua, J. 2010. Temperature modulates plant defense responses through NB-LRR proteins. PLoS Pathog. 6:e1000844. 